\title{
An Extended Maneuver Coordination Protocol with Support for Urban Scenarios and Mixed Traffic
}

\author{
Max Bastian Mertens ${ }^{1 \dagger}$, Johannes Müller ${ }^{1 \dagger}$, Robin Dehler ${ }^{1}$, \\ Marvin Klimke ${ }^{1,2}$, Matthias Maier ${ }^{3}$, Soheil Gherekhloo ${ }^{3}$, Benjamin Völz ${ }^{2}$, \\ Rüdiger-Walter Henn ${ }^{3}$, and Michael Buchholz ${ }^{1}$
}

\begin{abstract}
Coordinating the maneuvers between connected vehicles and other road users can increase traffic efficiency. To efficiently coordinate road users in a broad variety of maneuvers despite limited bandwidth, an appropriate message format and communication procedure must be defined. For this, first proposals have been made, mostly from a highway application perspective. However, we observe that many maneuvers that likely occur in urban scenarios are not covered by the existing proposals. Hence, in this work, we propose a new maneuver coordination message and a corresponding communication procedure with additional support for cooperative urban scenarios and mixed traffic of automated and manually driven vehicles. We illustrate the usage of our extended protocol with several example use cases.
\end{abstract}

\section{INTRODUCTION}

Coordinating the motion of connected vehicles and other road users offers a huge potential to further increase the traffic and fuel efficiency. However, to function on a large scale in real-world traffic across various platforms from different manufacturers, standardization is imperative. The European Telecommunications Standards Institute (ETSI) [1] standardizes messages and services for vehicle-to-anything (V2X) communication. The cooperative awareness message (CAM) [2] is a prominent result from this standardization. In turn, the standardization of a maneuver coordination scheme is still in its infancies [3]. Albeit first drafts have been proposed for the maneuver coordination message (MCM) [4]-[7], the existing proposals do not yet cover a sufficiently broad spectrum of application scenarios. Particularly, to the best of our knowledge, all the existing works do not consider centralized coordination schemes. The authors in [8] propose that one of the involved connected vehicles serves as a coordinator, in their scenario the vehicle closest to an intersection. However, in urban areas, edge computing resources can easily be provided, which can be used to efficiently coordinate cooperative road users.

This work was financially supported by the Federal Ministry of Economic Affairs and Energy of Germany within the program "Highly and Fully Automated Driving in Demanding Driving Situations" (project LUKAS, grant numbers 19A20004A and 19A20004F).

$\dagger$ M. Mertens and J. Müller are both first authors with equal contribution. E-mail addresses: \{max.mertens, johannes-christian.mueller\} Quni-ulm.de

${ }^{1}$ The authors are with the Institute of Measurement, Control and Microtechnology, Ulm University, D-89081 Ulm, Germany.

${ }^{2}$ The authors are with the Robert Bosch GmbH, Corporate Research, Renningen, Germany.

${ }^{3}$ The authors are with the Robert Bosch $\mathrm{GmbH}$, Cross-Domain Computing Solutions, Leonberg, Germany.
For connected and automated vehicles (CAV), a common approach is to send a desired trajectory in addition to their currently followed planned trajectory [5]. The desired trajectory is only feasible if other connected vehicles cooperate and accept the suggested maneuver or explicitly promise to follow a compatible trajectory [9]. In the survey [4], several decentralized approaches to cooperative driving are presented. With such approaches in mind, the ETSI started the standardization of the maneuver coordination service (MCS) and the corresponding MCM [3]. In the IMAGinE project, the message has been extended by a cost value representing how favorable the trajectories are for the sending vehicle [7]. The TransAID project [6] extended the MCM by a vehicle to infrastructure (V2I) interface, which assists in the negotiation of cooperative maneuvers. However, none of these proposals considered mixed traffic, i.e., automated and manually driven vehicles. The only MCM approach allowing for a centralized structure is [6], which only covers lane change maneuvers and speed advice. More complex scenarios, such as the coordination of junctions, are not considered.

In this paper, we extend the existing approaches and propose an MCM protocol with planning responsibility at a central coordinator. The coordinator can be a cooperating vehicle as proposed in [8], or a road side infrastructure server connected via ITS-G5 [1] or cellular network. We address mixed traffic with CAVs as well as connected nonautomated vehicles (CV) with a human-machine interface (HMI). The coordinator can additionally receive and process CAMs and collective perception messages (CPM) [10] sent by connected vehicles and infrastructure sensors. By generating a merged environment model, the coordinator can even consider perceived non-connected road users. Our approach can benefit from edge computing, as a stationary edge server can provide more computational power than a road user. Due to the centralized structure, information processing and coordination of maneuvers is performed at a single entity. Thus, bandwidth and computational resources are saved. We extend the scope of other MCM proposals by supporting highway use cases and adding cooperative urban scenarios to provide a widely applicable protocol.

The rest of this paper is structured as follows: first, we describe the MCM extension in Section II. Then, the communication procedure is sketched in Section III. Finally, we discuss our proposed extension with example use cases in Section IV and finish with conclusions in Section V. 


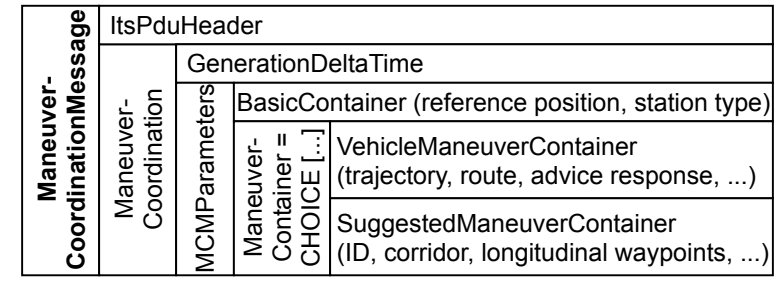

\begin{tabular}{|c|c|c|c|}
\hline \multirow{6}{*}{ 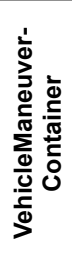 } & \multicolumn{3}{|c|}{ VehicleState (type, speed, yaw angle) } \\
\hline & \multirow{2}{*}{$\begin{array}{l}\text { Planned- } \\
\text { Trajectory }\end{array}$} & \multicolumn{2}{|c|}{ StartDeltaTime, DeltaTime } \\
\hline & & $\begin{array}{l}\text { List of } \\
\text { TrajectoryPoint }\end{array}$ & $\begin{array}{l}\text { XDistance, YDistance, } \\
\text { Speed, YawAngle }\end{array}$ \\
\hline & $\begin{array}{c}\text { Desired- } \\
\text { Route }\end{array}$ & List of Waypoint & XDistance, YDistance \\
\hline & Advice- & \multicolumn{2}{|c|}{ ManeuverID, AdviceUpdateID } \\
\hline & Response & \multicolumn{2}{|c|}{ AdviceFollowed (accept, reject, pending) } \\
\hline
\end{tabular}

\begin{tabular}{|c|c|c|c|c|}
\hline \multirow{11}{*}{ 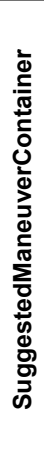 } & \multicolumn{4}{|c|}{ ManeuverID, AdviceUpdateID, TargetStationID } \\
\hline & \multicolumn{4}{|c|}{ ParticipatingRoadUserIDs } \\
\hline & \multicolumn{4}{|c|}{ ConfirmationRequiredFlag } \\
\hline & \multirow{8}{*}{ 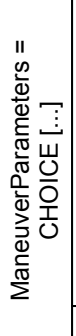 } & \multirow{7}{*}{ 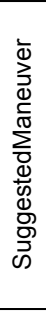 } & \multirow{6}{*}{$\begin{array}{l}\text { List of } \\
\text { Longitudinal- } \\
\text { Maneuver- } \\
\text { Waypoint }\end{array}$} & Waypoint (XDistance, YDistance) \\
\hline & & & & MinArrivalTime, MaxArrivalTime \\
\hline & & & & MinVelocity, MaxVelocity \\
\hline & & & & MinDurationOfStop \\
\hline & & & & $\begin{array}{l}\text { PrecedingRoadUserID, } \\
\text { FollowingRoadUserID }\end{array}$ \\
\hline & & & & YieldToRoadUserIDs \\
\hline & & & $\begin{array}{l}\text { Maneuver- } \\
\text { Corridor }\end{array}$ & Polygon: list of (XDist., YDist.) \\
\hline & & \multicolumn{3}{|c|}{ TerminationStatus: regular maneuver end or error } \\
\hline
\end{tabular}

Fig. 1. Proposed ManeuverCoordinationMessage and the included VehicleManeuverContainer and SuggestedManeuverContainer. The ItsPduHeader, GenerationDeltaTime and BasicContainer are the same as in the CAM [2]. Optional entries are highlighted in gray.

\section{Proposed Extension of the Maneuver COORDination Message}

An overview of our proposed MCM is given in Fig. 1. Like in [6], our MCM also comprises an ItsPduHeader and a ManeuverCoordination, which consists of a GenerationDeltaTime and the MCMParameters. The MCMParameters are further subdivided into a BasicContainer and a ManeuverContainer. Following the definition of the cooperative awareness message (CAM) [2], the BasicContainer comprises the reference position and the station type of the transmitting agent. In contrast, the definition of the ManeuverContainer differs from [6]. To follow the oneservice-one-message paradigm of the standard, the maneuver container is defined as a choice between a VehicleManeuverContainer or a SuggestedManeuverContainer, which is chosen according to the role of the communicating agent.

The VehicleManeuverContainer is transmitted frequently (e.g. with $1-10 \mathrm{~Hz}$ ) by CVs and CAVs willing to participate in cooperative maneuvers. It includes the vehicle state, the planned trajectory, the desired route, and a response to a received maneuver suggestion. The VehicleState comprises the type (CV or CAV), speed and yaw angle of a vehicle. The position is already given in the BasicContainer. The optional PlannedTrajectory applicable to CAVs comprises a list of trajectory points, where each contains a speed and yaw angle in addition to a waypoint to avoid differentiation of roughly discretized trajectories. The DesiredRoute contains a list of waypoints describing the route of the vehicle. The route has to be known to the coordinator but is mostly static, so it can be sent less frequently to reduce the average message size and thus is marked optional. The AdviceResponse refers to the ManeuverID and AdviceUpdateID of the suggested maneuver. It also contains the AdviceFollowed enumeration, which can take the states accept, reject, and pending, in case a vehicle has not yet confirmed or declined a request.

The SuggestedManeuverContainer is sent frequently (e.g. with $1-10 \mathrm{~Hz}$ ) by the coordinator to connected road users participating in a maneuver to broadcast trajectory proposals, update parameters, and provide a watchdog functionality. The recipient is indicated through the TargetStationID, while the incremental ManeuverID and AdviceUpdateID serve as a reference for the vehicles sending their agreement or disagreement. The ParticipatingRoadUserIDs lists all road users involved in the maneuver and can be used, e.g., to show a selective environment model on the HMI screen. An advice response by the addressee is only necessary if the respective ConfirmationRequiredFlag is set to true.

A SuggestedManeuver consists of a list of LongitudinalManeuverWaypoints used to encode velocity constraints. Each entry has a Waypoint it refers to, an arrival time interval MinArrivalTimestamp and MaxArrivalTimestamp, and optionally a velocity interval MinVelocity and MaxVelocity that the vehicle is allowed to have at the respective waypoint. To include mixed traffic scenarios, the optional MinDurationOfStop is particularly helpful. For example, a bus driver may set a stop duration that the bus will certainly need for the passengers to disembark. Furthermore, the coordinator may add the IDs of the road users, PrecedingRoadUserID and FollowingRoadUserID, between which the addressed vehicle is intended to drive, to assist in gap synchronization. Additionally, a list YieldToRoadUserIDs is included, indicating the road users relevant to this longitudinal waypoint as described in Section III-D. Finally, an optional ManeuverCorridor, a list of coordinates, i.e. XDistance and YDistance, can be transmitted. It describes a polygon for the lateral constraints and is only applicable to connected automated vehicles. When a maneuver ends regularly or abnormally, a TerminationStatus is sent instead of a SuggestedManeuver.

The IDs in the SuggestedManeuverContainer referring to other road users are provided as additional information for a more efficient trajectory planning or a more comprehensive HMI display. Thus, the existence of a collective perception is not essential for the proposed MCM protocol. However, to efficiently use this information, the coordinator can additionally broadcast an environment model via CPM containing the road users that the respective IDs are referring to.

To avoid ambiguity, the coordinator needs to set the object IDs of perceived connected road users to their station IDs. The ETSI communications security architecture [11] proposes frequently changing station IDs to achieve pseudonymity. Therefore, if this is implemented, the Lock ID Change service in [11] has to be used to postpone station ID changes until the maneuver has been completed. 


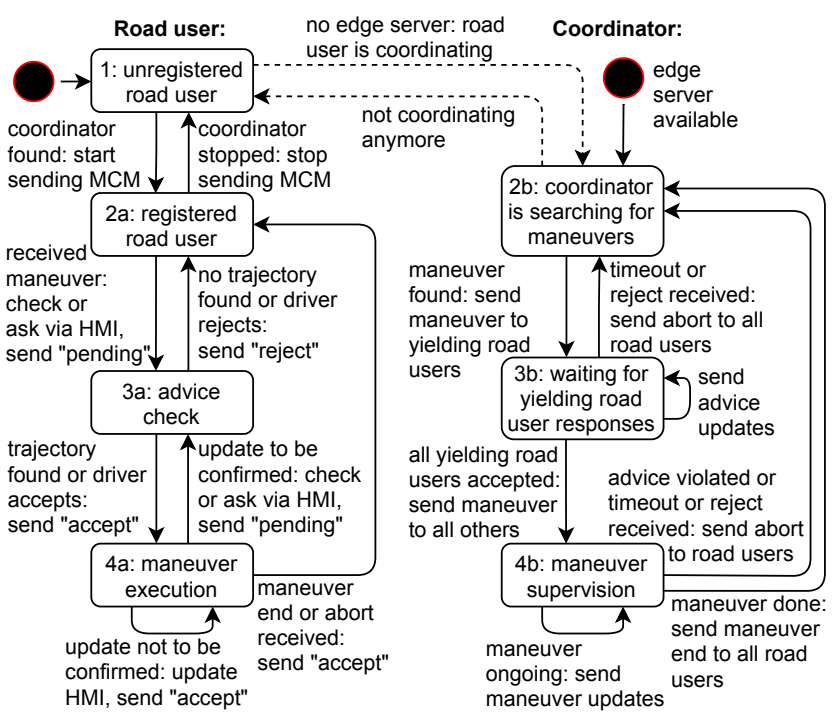

(a) MCM protocol state diagram.

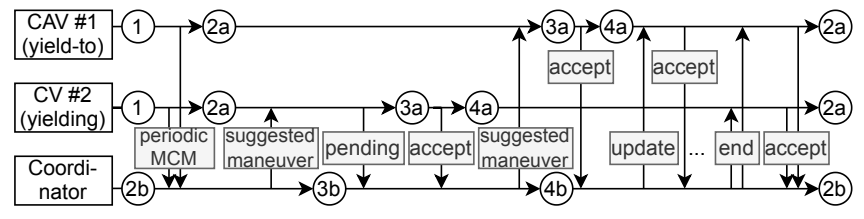

(b) Exemplary MCM protocol sequence diagram with respective states. In the suggested maneuver, CAV \#1 is prioritized, while CV \#2 should yield.

Fig. 2. Suggested MCM protocol.

\section{Communication Procedure}

An overview of the communication protocol is shown in Fig. 2a and explained in Sections III-A to III-C, with an example sequence diagram in Fig. 2b. A central coordinator has to be determined before maneuver coordination is possible. In case a road side infrastructure with a local server is available (known by a previously shared list of servers and corresponding governed regions), this edge server is handling the coordination. In autonomous scenarios without an edge server, a connected road user can initiate cooperative scenarios by serving as a coordinator, as described in [8].

\section{A. Road user registration and maneuver preparation}

Cooperative road users have to register at the coordinator to announce their presence and cooperation by regularly sending the VehicleManeuverContainer (stage 2a in Fig. 2). The frequency is chosen such that the expected errors remain tolerable. The planning module of the coordinator constantly analyzes the traffic scenario and determines if a cooperative maneuver could significantly improve traffic efficiency (stage 2b). As soon as such a maneuver is found, the respective corridors, trajectories, and intervals are planned and sent to the participating road users as described in Section III-C.

\section{B. Maneuver execution as a road user}

A road user participating in cooperative maneuvers receives SuggestedManeuverContainers with a maneuver advice (stage 3a in Fig. 2). In case of a CAV, the planning module tries to plan a compatible trajectory and reports the result, whereas in a manually driven connected vehicle, the driver is asked for confirmation via the HMI. Until acceptance or rejection, a pending decision is sent to the coordinator. The lateral corridor and longitudinal intervals are chosen so that driving within those bounds is guaranteed to be safe, assuming typical physical bounds and adherence to accepted maneuvers of all road users.

After a road user accepted a suggestion (stage 4a), it executes the maneuver and can receive two types of maneuver updates. Updates with additional restrictions such as longer waiting times or lower velocities are marked by a flag and need another confirmation by the driver or planning module. Until confirmation, the decision is reset to a pending state. In turn, trivial updates with loosened constraints are automatically accepted without the need for confirmation. When no parameter is changed, the same maneuver with the ConfirmationRequiredFlag set to false is retransmitted.

\section{Maneuver coordination}

Most cooperative scenarios have a common structure (see Section IV): A different driving or crossing order than implied by the traffic rules is imposed on the participating vehicles. Some road users are prioritized, while others should yield to improve the overall traffic efficiency. Note that yielding is meant in a broader way and refers to slowing down or stopping to let other road users merge, cross, or pass. Therefore, a cooperative maneuver is considered to have a two-stage structure. To ensure safety, first, all yielding road users have to accept the suggested maneuver (stage $3 \mathrm{~b}$ in Fig. 2). Afterwards, the prioritized road users are free to drive and can be given temporary right of way, encoded by the lateral corridor and longitudinal intervals (stage 4b). More complex dependencies between vehicles can be realized by additional negotiation stages. Until the end of the maneuver, regular parameter updates are sent to the participating road users to reflect a changed situation. To handle packet loss, a maneuver suggestion is re-transmitted until a response is received. A vehicle not responding for a certain time is assumed to reject the maneuver. When a suggestion is rejected or an accepted maneuver is violated at any time, the whole maneuver is aborted by the coordinator. In this case, the road users should fall back to regular driving and reach a traffic rule conform state as quickly as possible.

\section{Preliminary constraint removal}

Longitudinal constraints sent in a suggested maneuver are calculated with margins large enough to guarantee safety. Sometimes the constraining intervals are underapproximated. To retain traffic efficiency, a list of yield-to road user IDs can be sent, giving the road users relevant to the restriction. As soon as all included road users have become irrelevant (e.g., left the junction), the LongitudinalManeuverWaypoint can be disregarded and the yielding vehicle can drive on.

\section{Use CASES}

To illustrate the broad applicability of our MCM extension proposal, the scenarios a) - e) shown in Fig. 3 are presented 


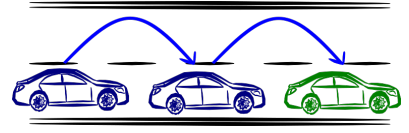

a) CACC scenario

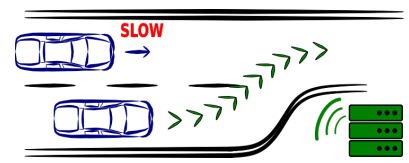

b) Lane change (ending lane)

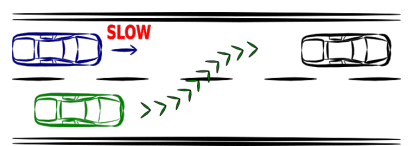

c) Lane change scenario

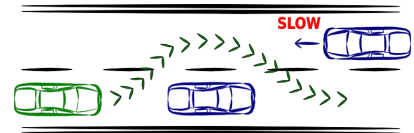

d) Overtaking scenario

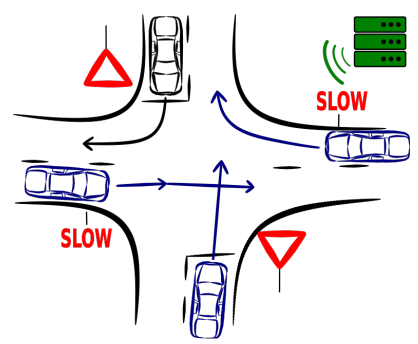

e) Complex junction
Fig. 3. Example scenarios for the MCM usage. Green: coordinating vehicle or edge server; blue: connected vehicle; black: non-connected vehicle.

exemplarily. In all use cases, an edge server can be used for coordination. Some exemplary scenarios are shown where no edge server is available and the green vehicle serves as the coordinator. Sample messages were encoded in ASN.1 as proposed in [1]. The size of messages from the coordinator ranges from $57 \mathrm{~B}$ to $109 \mathrm{~B}$ in the shown use cases. Message sizes in the other direction depend on the trajectory and route granularity and reach $570 \mathrm{~B}$ for 60 trajectory points.

To show that our proposal handles existing cooperative use cases, the first scenario a) in Fig. 3 describes a Cooperative Adaptive Cruise Control (CACC) [12] situation, in which the leading CAV coordinates. The DesiredRoute indicates how long vehicles will stay in the platoon. The lateral corridor is not required, as it is given by the lane boundaries. Since CACC is mostly reactive, the LongitudinalManeuverWaypoints can contain only two elements. The PrecedingRoadUserID provides the predecessor of each CAV. The time and velocity intervals are broadcasted to all participants and allow for predictive CACC.

In scenario b), the coordination is performed by an edge server. The vehicle on the left lane needs to yield to ensure safety. The right vehicle is given a ManeuverCorridor polygon of seven waypoints describing the lane end and one LongitudinalWaypoint with a MaxArrivalTime and the left vehicle as a FollowingRoadUserID.

Scenario c) shows a lane change maneuver, where the coordinating vehicle requests the blue vehicle to slow down. For this, it sends one LongitudinalWaypoint and itself as the yield-to road user. After the blue vehicle accepts to yield, the green vehicle can safely change the lane.

In scenario d), for safe overtaking, the coordinating vehicle requests an oncoming $\mathrm{CV}$ to slow down. The overtaken $\mathrm{CV}$ could be a post van or dustcart that already frequently stops in urban scenarios. It is requested to stop at the curb with a MinDurationOfStop. The overtaking vehicle is given as the yield-to road user, so the stopping vehicle can already drive on if the overtaking is completed before the stop duration is over. Requesting a maximum velocity instead of a stop is an alternative to overtake slow vehicles, such as street cleaners.
Finally, scenario e) shows a junction in which the coordinator proposes the overall most favorable (e.g. energy efficient) maneuver, with one or two LongitudinalWaypoints and respective YieldToRoadUserIDs for each yielding vehicle.

\section{CONClusion And Future Work}

In this paper, we presented an extension of existing MCM format proposals, adding urban scenarios to the use cases addressed in the cited previous works. The various scenarios are handled even in mixed traffic by coordinating connected automated and non-automated vehicles. Our extension additionally supports centralized structures, e.g., road side infrastructure with edge servers. We hope that our proposal can contribute to the ETSI MCM standardization efforts. In our ongoing work, we use the proposed MCM for edge server-based planning and coordination of connected road users to increase efficiency in cooperative urban scenarios. On a pilot site in real traffic [13], besides a proof-of-concept, we plan to evaluate also the communication protocol with respect to aspects like latency and reliability.

\section{REFERENCES}

[1] ETSI ES 202 663, “Intelligent Transport Systems (ITS); European profile standard for the physical and medium access control layer of Intelligent Transport Systems operating in the $5 \mathrm{GHz}$ frequency band," Nov. 2009, final draft V1.1.0.

[2] ETSI EN 302 637-2, "Intelligent Transport Systems (ITS); Vehicular Communications; Basic Set of Applications; Part 2: Specification of Cooperative Awareness Basic Service,” Sep. 2014, final draft V1.3.1.

[3] ETSI TR 103 578, "Intelligent Transport Systems (ITS); Vehicular Communication; Informative Report for the Maneuver Coordination Service," May 2020, draft V0.0.5.

[4] D. Maksimovski, A. Festag, and C. Facchi, "A Survey on Decentralized Cooperative Maneuver Coordination for Connected and Automated Vehicles," in Proc. Int. Conf. Veh. Tech. Intell. Transp. Syst. - (VEHITS), 2021, pp. 100-111.

[5] B. Lehmann, H.-J. Günther, and L. Wolf, "A Generic Approach towards Maneuver Coordination for Automated Vehicles," in Proc. IEEE Int. Conf. Intell. Transp. Syst. (ITSC), Nov. 2018, p. 3333.

[6] A. Correa, R. Alms, J. Gozalvez, M. Sepulcre, M. Rondinone, R. Blokpoel, L. Lücken, and G. Thandavarayan, "Infrastructure Support for Cooperative Maneuvers in Connected and Automated Driving," in Proc. IEEE Intell. Vehicles Symp. (IV), Jun. 2019, pp. 20-25.

[7] I. Llatser, T. Michalke, M. Dolgov, F. Wildschütte, and H. Fuchs, "Cooperative Automated Driving Use Cases for 5G V2X Communication," in IEEE $5 G$ World Forum (5GWF), Sep. 2019, pp. 120-125.

[8] A. A. Hassan and H. A. Rakha, "A Fully-Distributed Heuristic Algorithm for Control of Autonomous Vehicle Movements at Isolated Intersections," Int. Jour. of Transp. Science Tech., vol. 3, no. 4, pp. 297-309, Dec. 2014.

[9] W. Xu, A. Willecke, M. Wegner, L. Wolf, and R. Kapitza, "Autonomous Maneuver Coordination Via Vehicular Communication," in Proc. Annu. IEEE/IFIP Int. Conf. Dependable Sys. and Netw. Workshops (DSN-W), Jun. 2019, pp. 70-77.

[10] ETSI TR 103 562, "Intelligent Transport Systems (ITS); Vehicular Communications; Basic Set of Applications; Analysis of the Collective Perception Service (CPS); Release 2," Dec. 2019, v2.1.1.

[11] ETSI TS 102 940, "Intelligent Transport Systems (ITS); Security; ITS communications security architecture and security management," Apr. 2018, v1.3.1.

[12] Z. Wang, G. Wu, and M. J. Barth, "A Review on Cooperative Adaptive Cruise Control (CACC) Systems: Architectures, Controls, and Applications," in Proc. IEEE Int. Conf. Intell. Transp. Syst. (ITSC), Nov. 2018, pp. 2884-2891.

[13] M. Buchholz, J. C. Müller, M. Herrmann, J. Strohbeck, B. Völz, M. Maier, J. Paczia, O. Stein, H. Rehborn, and R.-W. Henn, "Handling Occlusions in Automated Driving Using a Multiaccess Edge Computing Server-Based Environment Model From Infrastructure Sensors," IEEE Intell. Transp. Syst. Mag., 2021. 